\title{
How many key informants are enough? Analysing the validity of the community readiness assessment
}

\author{
Saskia Muellmann ${ }^{1}$, Tilman Brand ${ }^{1}$, Dorothee Jürgens ${ }^{1}$, Dirk Gansefort ${ }^{1,2}$ and Hajo Zeeb ${ }^{1,3^{*}}$ (c)
}

\begin{abstract}
Objective: Communities are important settings for health promotion and prevention. The community readiness assessment offers a structured approach to assess resources and opportunities to tackle a health problem within a community. The assessment relies on semi-structured interviews with key informants from the communities. A number of 4-6 key informant interviews are recommended in the literature. However, it is unclear whether this is sufficient to obtain a valid representation of the respective community. This study analysed whether increasing the number of key informants from 4-6 to 12-15 alters the results of the community readiness assessment.

Results: A total of 55 community readiness interviews were carried out in 4 communities. Overall, the community readiness scores showed little variation after having interviewed 10 key informants in a community. However, even after completing 10 interviews in a community, key informants were still able to identify up to 6 new information items regarding community efforts for physical activity promotion among the elderly, contact and communication channel for informing or approaching the target group, or barriers to participation.
\end{abstract}

Keywords: Community-based health promotion, Assessment, Community readiness, Validity, Key informants

\section{Introduction}

Community-based interventions are important approaches to prevention and health promotion because they can reach their target population in their natural living environment [1]. Such interventions can initiate changes not only on the individual level but also on different levels of the social and built environment and thus may lead to sustainable health improvements [2]. While the term community covers a broad range of social groups that share a common identity or other characteristics [3], in this article community refers to small scale geographical areas where people live under the same

\footnotetext{
*Correspondence: zeeb@bips.uni-bremen.de

${ }^{1}$ Department of Prevention and Evaluation, Leibniz-Institute for Prevention Research and Epidemiology - BIPS, Achterstr 30, 28359 Bremen, Germany

Full list of author information is available at the end of the article
}

political administration such as neighbourhoods, city districts or towns. Community-based approaches often rely on early involvement of local stakeholders and residents to foster participation and capacity building [4]. The community readiness assessment (CRA) offers a structured approach to assess resources and opportunities to tackle a health problem within a community [5]. It builds upon a stages-of-change approach and associates each community with a certain degree of preparedness ranging from no awareness to professionalization of efforts. The assessment relies on semi-structured interviews with key informants from the communities. A number of 4-6 key informant interviews are recommended in the community readiness $(\mathrm{CR})$ literature $[5,6]$. However, it is unclear whether this number suffices to obtain a valid representation of the community [7]. In qualitative research, the concept of data saturation refers to the point in data collection when no new information arises from subsequent

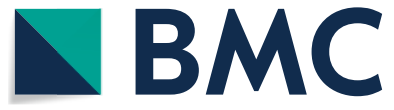

(c) The Author(s) 2021. This article is licensed under a Creative Commons Attribution 4.0 International License, which permits use, sharing, adaptation, distribution and reproduction in any medium or format, as long as you give appropriate credit to the original author(s) and the source, provide a link to the Creative Commons licence, and indicate if changes were made. The images or other third party material in this article are included in the article's Creative Commons licence, unless indicated otherwise in a credit line to the material. If material is not included in the article's Creative Commons licence and your intended use is not permitted by statutory regulation or exceeds the permitted use, you will need to obtain permission directly from the copyright holder. To view a copy of this licence, visit http://creativeco mmons.org/licenses/by/4.0/. The Creative Commons Public Domain Dedication waiver (http://creativecommons.org/publicdomain/ zero/1.0/) applies to the data made available in this article, unless otherwise stated in a credit line to the data. 
data collection efforts $[8,9]$. Studies examining the number of qualitative interviews required to reach data saturation found that 12 [10] to 17 interviews [8] appeared to be sufficient. Nevertheless, the number of interviews necessary to achieve a valid CR assessment in the field of physical activity (PA) promotion has not been investigated yet. As part of a community-based research project on PA promotion for the elderly (AEQUIPA - Ready to Change) [11], this study analysed whether increasing the number of key informants from 4-6 to $12-15$ alters the results of the CRA.

\section{Main text \\ Methods \\ Study population}

Key informants (e.g., representatives from local public authorities, senior citizen advocacy groups, sports clubs) were either identified via online searches or recommended by other key informants. They were interviewed with an adapted version of the CRA targeting PA in older adults $[12,13]$. The Ready to Change project entailed several rounds of CRA in the participating communities. In the first round in 2015, 4-6 key informants per community were interviewed. In the second round in 2018 (data base of this analysis), the key informants from the first round were interviewed again, but the sample was extended by 6-10 new key informants in each community.

\section{Data collection}

The CRA was conducted between April and May 2018 in four communities in the Metropolitan region Bremen-Oldenburg, Germany. The sample comprised two semi-rural (both about 30,000 inhabitants) and two urban communities (districts of a larger city; 27,000 and 36,000 inhabitants, respectively). The communities were selected based on the fact they witnessed a marked increase in population ageing. Levels of deprivation and sociocultural diversity were higher in the urban communities compared to the semi-rural ones. The adapted CRA interview guide contained closed and open-ended questions addressing the five dimensions of the CR [5]: 1. Community efforts and knowledge of efforts, 2 . Leadership, 3. Community climate, 4 . Community knowledge of the issue, and 5. Resources. Interviews were conducted face-to-face or via telephone. All interviews were audiotaped and transcribed verbatim.

\section{Analysis}

Based on the instructions of the CR manual [5], each interview was scored independently by two researchers to provide a CR score for each dimension and an overall CR score using a nine-point rating scale (scores between
$1=$ no awareness and $9=$ professionalization). To examine whether increasing the number of interviews from initially 4-6 to $12-15$ leads to a change in the CR score, the mean CR score of the initial sample was compared with the mean CR score of the newly identified key informants for each community. Mean differences were calculated with 95\% confidence intervals (CI). For analyzing whether mean $C R$ scores stabilize with increasing numbers of interviews, change in the mean CR score was calculated for each new key informant interviewed. Prior to the analysis, we defined that the mean CR score is stable if the change with every new respondent is $\leq 0.1$ standard deviations (SD), taking Cohen's classification of effect sizes as an orientation [14]. Therefore, we calculated change in the $\mathrm{CR}$ score as standardized mean differences (Standardized mean difference $=(C R$ mean score $_{(x}$ respondents +1$)-C R$ mean score ${ }_{(x}$ respondents) $) / S D{ }_{(C R}$ mean score). . The analysis of the stabilization of the mean CR scores was performed using the original order of conducted interviews and a random order of respondents. In addition to the analysis of the CR score, we wanted to find out the level of content-related saturation that was reached by increasing the number of interviews. To this end, we selected three open-ended questions from the $\mathrm{CR}$ interview guide and analysed whether an interview added new information regarding (a) community efforts for PA promotion among the elderly, (b) contact and communication channel for informing or approaching the target group, and (c) barriers to participation. A linear regression was performed to analyse the association between the number of interviews and newly identified issues of the CR.

\section{Results}

A total of 55 respondents were interviewed. The number of interviews per community varied from 12 to 15 . The majority of key informants were from sports clubs or sports facilities $(40 \%)$ or worked for public authorities (36\%). 15\% were representatives from senior citizen advocacy groups and 9\% worked for civil services in the communities. The mean age of the respondents was 59 years (standard deviation $=9.25$, range: $29-85$ ). $56 \%$ of the respondents were female and $44 \%$ were male. At some point during the recruitment process it appeared difficult to identify further key informants in some of the communities. This is the reason why it was not possible to obtain 15 interviews in all communities.

A comparison of the CR scores from the initial sample with the extended sample revealed mean differences of -0.45 to $0.67 \mathrm{CR}$ scale points across the communities (Table 1). None of the mean differences was statistically significant. 
Table 1 Comparison of CR score in the standard and the extended sample by community

\begin{tabular}{|c|c|c|c|c|}
\hline \multirow[t]{2}{*}{ Community } & \multicolumn{3}{|c|}{ Community readiness score } & \multirow{2}{*}{$\begin{array}{l}\text { Mean differences } \\
\text { (extended-initial) (95\% } \\
\text { Cl) }\end{array}$} \\
\hline & Initial sample & Extended sample & Combined & \\
\hline Community A & $4.51(0.72 ; n=5)$ & $5.18(0.82 ; n=10)$ & $4.96(0.83 ; n=15)$ & $0.67(-0.27-1.61)$ \\
\hline Community B & $4.96(0.66 ; n=5)$ & $4.72(0.60 ; n=9)$ & $4.81(0.61 ; n=14)$ & $-0.24(-1.00-0.51)$ \\
\hline Community $\mathrm{C}$ & $4.97(0.62 ; n=5)$ & $4.52(0.66 ; n=7)$ & $4.71(0.65 ; n=12)$ & $-0.45(-1.29-0.38)$ \\
\hline Community D & $4.92(0.51 ; n=5)$ & $4.69(0.47 ; n=9)$ & $4.78(0.48 ; n=14)$ & $-0.23(-0.84-0.37)$ \\
\hline
\end{tabular}

Numbers are means with standard deviations in brackets

Figure 1 shows how the CR score changed with increasing number of interviews. Overall, the figure indicates a convergence of the CR scores with only little variation after having interviewed 9 or 10 key informants.

Figure 2 displays the change in the CR score in the four communities when adding more interviews as standardized mean differences. After interviewing 10 key informants the change in the CR is smaller than $\pm 10 \%$ of the $\mathrm{SD}$ which translates into $0.08 \mathrm{CR}$ scale points. Using a random order of respondents did not change the results suggesting that after interviewing 10 key informants little variation in the $\mathrm{CR}$ scores can be observed (Additional file 1: Figure S1 and S2).

Overall, a linear regression analysis confirmed a decline in newly identified issues with increasing number of interviews, although this association did not pass the threshold for statistical significance in the case of community efforts (Additional file 1: Table S1). Thus, there is a trend that with every additional interview the amount of new information gained from it becomes smaller. However, as can be seen in the (Additional file 1: Figure
S3 to S5), even after completing 10 interviews in a community, respondents were still able to identify up to 6 new community efforts for PA promotion.

\section{Discussion}

This study aimed to analyse whether increasing the number of key informants from 4-6 to 12-15 alters the results of the CRA. Overall, there were no large differences in the CR scores between the initial and the extended sample. Extending the sample did not change the mapping of the communities to a certain transtheoretical stage of change in the CR model. The CR scores showed little variation after having interviewed 10 key informants in a community, indicating that this may be a good number to strive for in CRA interviews. Our results are similar to a study by Guest et al. [10] who systematically examined the degree of data saturation in 60 in-depth interviews with women in two African countries. The authors started with analysing the first six interviews, then added a set of six more interviews, and so on. The process was repeated until all 60 interviews have been analysed. The

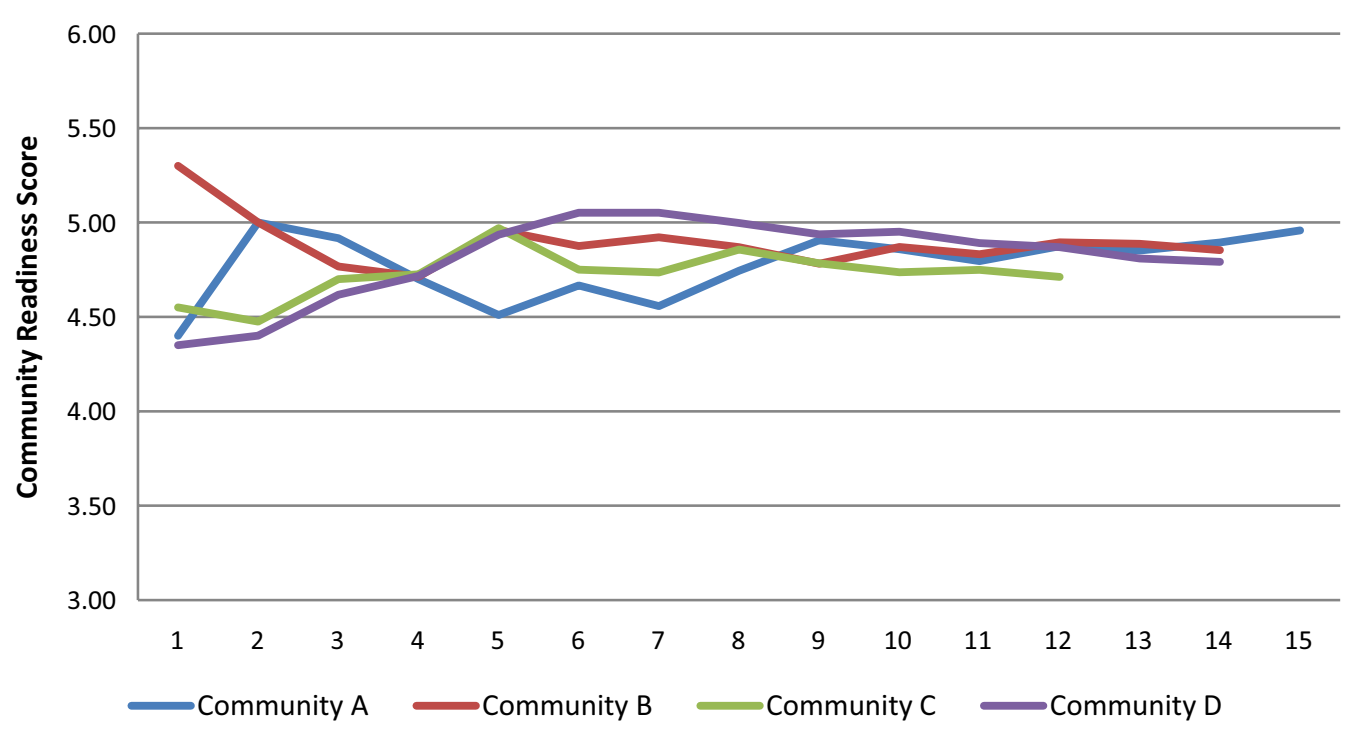

Fig. 1 Mean CR score in 4 communities. Number of respondents are shown on the $X$ axis 


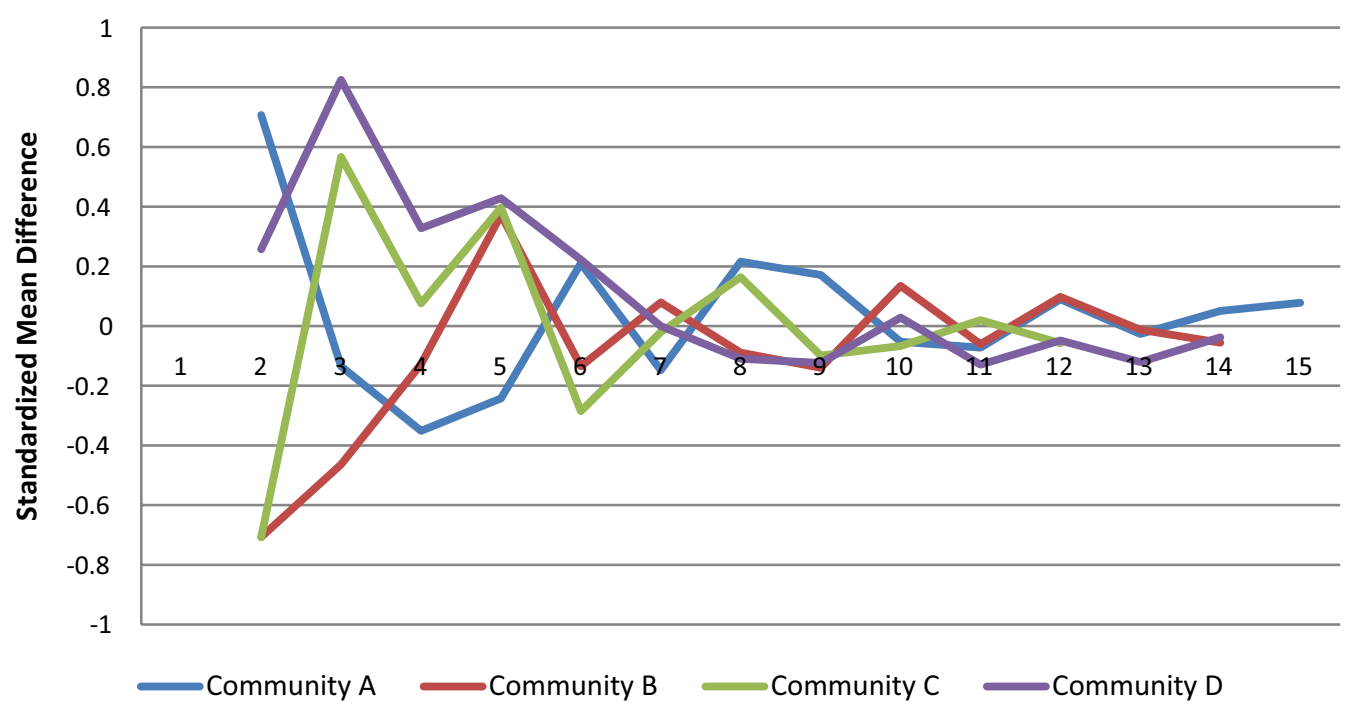

Fig. 2 Standardized change in CR mean score over number of respondents ( $X$ axis)

authors found that data saturation occurred within the first 12 interviews with $92 \%$ of codes identified therein [10]. Two approaches on data saturation, code saturation (i.e., no additional issues raised, codebook stabilized) and meaning saturation (i.e., no further nuances or dimensions can be found), were examined in 25 in-depth interviews with HIV patients [9]. The authors found that code saturation was reached after nine interviews (91\% of codes identified) and meaning saturation was achieved between 16 and 24 interviews. In addition, a study by Francis et al. [8] found that data saturation (i.e., minimum of 10 interviews, no new themes after analysing 3 further interviews) in theory-based interview studies was achieved after conducting 17 interviews. Focusing on studies applying the CR tool, Kostadinov et al. [6] included 40 articles published between 1999 and 2013 in a systematic review. The authors reported that the number of interviews per community varied between one and 33 (mean $=7.3, \mathrm{SD}=5.9$, median $=6$, six studies provided no information on the number of interviews per community), with $15 \%$ of studies using less than the recommended four interviews per community. This is in line with studies published since 2013 that followed the recommendations of the $C R$ handbook and conducted an average of four to eight interviews per community (e.g., in [15-19]). However, Kesten et al. [20] used the concept of theoretical saturation (i.e., no new concepts are expected by conducting more interviews) to determine the necessary number of interviews for assessing CR for overweight and obesity prevention in pre-adolescent girls. Theoretical saturation was reached after conducting 33 interviews with key informants (e.g., parents, teachers).
In addition, Pradeilles et al. [21] interviewed 12 key informants per community across two cities in Ghana, but did not give any reasons for selecting 12 interviewees per community. While it seems that conducting 10 interviews per community provides a fairly stable classification of a community in terms of their CR score, our results also indicate that there may still be unidentified issues regarding community efforts for PA promotion, contact and communication channel for informing or approaching the target group, or barriers to participation in PA promotion. A reason for this could be the nature of the PA efforts in the communities. There were many small scattered efforts and thus it appeared that the key informants did not mention all or were not aware of them. This indicates the context- and topic-relatedness of CRA, which needs to be kept in mind when planning similar studies.

\section{Limitations}

Assuming stability of a mean if the change is less than 0.1 SD with every new respondent is an arbitrary choice. One could argue that small differences can lead to large changes if the sample is large and the differences go into the same direction. However, the results in our four communities did not indicate such a monotone pattern concerning the direction of change. Furthermore, there is a natural limit to the sample size of key informants. The number of potential key informants depends on the topic, the existing structures in the community, and the size of the community. For instance, in one of our selected communities it was not possible to identify more than 12 key informants who 
could provide information on community-based PA promotion in older adults. Our sampling strategy has the limitation that if key respondents recommend other potential key respondents (snowballing) the observations may not be independent from each other because the respondents may have shared views and attitude concerning the topic. Therefore we did not solely rely on snowballing but performed also internet searches to identify respondents. Another limitation is that in some cases key informants answered from their own point of view and may have had difficulties to respond on behalf of their community.

\section{Abbreviations}

$\mathrm{Cl}$ : Confidence interval; CR: Community readiness; CRA: Community readiness assessment; PA: Physical activity; SD: Standard deviation.

\section{Supplementary Information}

The online version contains supplementary material available at https://doi. org/10.1186/s13104-021-05497-9.

Additional file 1: Figure S1. Mean CR score in 4 communities with random order of respondents. Number of participants are shown on the $X$ axis. Figure $\mathbf{S 2}$. Standardized change in CR mean score over number of respondents ( $X$ axis) with random order of respondents. Table S1. Association between number of interviews and newly identified issues of CR (linear ordinary least square regression). Figure S3. Newly identified community efforts for the promotion of physical activity for older adults by number of key informants (X axis). Figure S4. Newly identified contact and information channels for the promotion of physical activity for older adults by number of key informants (X axis). Figure S5. Newly identified barriers to participation in physical activity for older adults by number of key informants ( $X$ axis).

\section{Acknowledgements}

Not applicable.

\section{Authors' contributions}

SM and TB drafted the manuscript. TB analysed the data. TB and HZ conceived and designed the study. DJ and DG scored the interviews. All authors read and approved the final version of the manuscript.

\section{Funding}

Open Access funding enabled and organized by Projekt DEAL. This study was funded by the German Federal Ministry of Education and Research (BMBF; Grant Number 01EL1822A). The content of this article only reflects the authors' views and the funder is not liable for any use that may be made of the information contained therein.

\section{Availability of data and materials}

The data that support the findings of this study are available from the corresponding author on reasonable request.

\section{Declarations}

\section{Ethics approval and consent to participate}

This study was approved by the Ethics Committee of the University of Bremen, Germany, in April 2018. All interviewees received written information about the study and gave informed consent for their data to be used.

\section{Consent for publication}

Not applicable.

\section{Competing interests}

All authors declare no conflict of interest.

\section{Author details}

${ }^{1}$ Department of Prevention and Evaluation, Leibniz-Institute for Prevention Research and Epidemiology - BIPS, Achterstr 30, 28359 Bremen, Germany.

${ }^{2}$ Association for Health Promotion and Academy of Social Medicine Lower Saxony, Fenskenweg 2, 30165 Hannover, Germany. ${ }^{3}$ Health Sciences Bremen, University of Bremen, Bremen, Germany.

Received: 16 September 2020 Accepted: 23 February 2021

Published online: 09 March 2021

\section{References}

1. World Health Organization. The Ottawa Charter for Health Promotion. Geneva: World Health Organization; 1986.

2. Sallis JF, Cervero RB, Ascher W, Henderson KA, Kraft MK, Kerr J. An ecological approach to creating active living communities. Annu Rev Public Health. 2006;27:297-322.

3. World Health Organization. Health Promotion Glossary. Geneva: World Health Organization; 1998.

4. Bopp M, Fallon E. Community-based interventions to promote increased physical activity: a primer. Appl Health Econ Health Policy. 2008;6(4):173-87.

5. Stanley L. Community readiness for community change. 2nd ed. TriEthnic Center for Prevention Research: Fort Collins; 2014.

6. Kostadinov I, Daniel M, Stanley L, Gancia A, Cargo M. A systematic review of community readiness tool applications: implications for reporting. Int J Environ Res Public Health. 2015;12(4):3453-68.

7. Kesten JM, Griffiths PL, Cameron N. A critical discussion of the Community Readiness Model using a case study of childhood obesity prevention in England. Health Soc Care Community. 2015;23(3):262-71.

8. Francis JJ, Johnston M, Robertson C, Glidewell L, Entwistle V, Eccles MP, Grimshaw JM. What is an adequate sample size? Operationalising data saturation for theory-based interview studies. Psychol Health. 2010;25(10):1229-45.

9. Hennink MM, Kaiser BN, Marconi VC. Code saturation versus meaning saturation: how many interviews are enough? Qual Health Res. 2017;27(4):591-608.

10. Guest B, Bunce A, Johnson L. How many interviews are enough? An experiment with data saturation and variablility. Field Methods. 2006;18(1):59-82.

11. Brand T, Gansefort D, Rothgang H, Roseler S, Meyer J, Zeeb H. Promoting community readiness for physical activity among older adults in Germany - protocol of the ready to change intervention trial. BMC Public Health. 2016;16:99.

12. Gansefort D, Peters M, Brand T. Wie bereit ist die Kommune? Das Community Readiness-Modell und die beispielhafte Anwednung in der kommunalen Gesundheitsförderung. Gesundheitswesen. 2020. Accessed 28th April).

13. Gansefort D, Brand T, Princk C, Zeeb H. Community readiness for the promotion of physical activity in older adults-a cross-sectional comparison of rural and urban communities. Int J Environ Res Public Health. 2018;15(3):453

14. Cohen J. Statistical power analysis for the behavioral sciences. 2nd ed New York: Lawrance Erlbaum Associates; 1988.

15. Islam S, Small N, Bryant M, Bridges S, Hancock N, Dickerson J. Assessing community readiness for early intervention programmes to promote social and emotional health in children. Health Expect. 2019;22(3):575-84

16. Liu M, Zhang X, Xiao J, Ge F, Tang S, Belza B. Community readiness assessment for disseminating evidence-based physical activity programs to older adults in Changsha, China: a case for Enhance(R)Fitness. Glob Health Promot. 2020;27(1):59-67.

17. Anderson-Carpenter KD, Watson-Thompson J, Jones MD, Chaney L. Improving community readiness for change through coalition capacity building: evidence from a multi-site intervention. J Community Psychol. 2017;45(4):486-99. 
18. Wichmann F, Braun M, Ganz T, Lubasch J, Heidenreich T, Laging M, Pischke CR. Assessment of campus community readiness for tailoring implementation of evidence-based online programs to prevent risky substance use among university students in Germany. Transl Behav Med. 2020;10(1):114-22.

19. Whelan J, Love P, Millar L, Allender S, Morley C, Bell C. A rural community moves closer to sustainable obesity prevention - an exploration of community readiness pre and post a community-based participatory intervention. BMC Public Health. 2019;19(1):1420.

20. Kesten JM, Cameron N, Griffiths PL. Assessing community readiness for overweight and obesity prevention in pre-adolescent girls: a case study. BMC Public Health. 2013;13:1205.
21. Pradeilles R, Marr C, Laar A, Holdsworth M, Zotor F, Tandoh A, Klomegah S, Coleman N, Bash K, Green M, et al. How ready are communities to implement actions to improve diets of adolescent girls and women in urban Ghana? BMC Public Health. 2019;19(1):646.

\section{Publisher's Note}

Springer Nature remains neutral with regard to jurisdictional claims in published maps and institutional affiliations.
Ready to submit your research? Choose BMC and benefit from:

- fast, convenient online submission

- thorough peer review by experienced researchers in your field

- rapid publication on acceptance

- support for research data, including large and complex data types

- gold Open Access which fosters wider collaboration and increased citations

- maximum visibility for your research: over 100M website views per year

At BMC, research is always in progress.

Learn more biomedcentral.com/submissions 